\title{
Strong light scattering in macroporous TiO2 monoliths induced by phase separation
}

\author{
$\operatorname{AUTHOR}(\mathrm{S})$ :
}

Fujita, K; Konishi, J; Nakanishi, K; Hirao, K

\section{CITATION:}

Fujita, K ... [et al]. Strong light scattering in macroporous TiO2 monoliths induced by phase separation. APPLIED PHYSICS LETTERS 2004, 85(23): 5595-5597

\section{ISSUE DATE:}

2004-12-06

URL:

http://hdl.handle.net/2433/50196

\section{RIGHT:}

Copyright 2004 American Institute of Physics. This article may be downloaded for personal use only. Any other use requires prior permission of the author and the American Institute of Physics. 


\title{
Strong light scattering in macroporous $\mathrm{TiO}_{2}$ monoliths induced by phase separation
}

\author{
Koji Fujita, ${ }^{a}$ Junko Konishi, Kazuki Nakanishi, and Kazuyuki Hirao \\ Department of Material Chemistry, Graduate School of Engineering, Kyoto University, Kyotodaigaku- \\ katsura, Nishikyo-ku, Kyoto 615-8510, Japan
}

(Received 10 May 2004; accepted 17 September 2004)

\begin{abstract}
Macroporous titania $\left(\mathrm{TiO}_{2}\right)$ monoliths have been prepared from systems containing titania colloid and poly(ethylene oxide) using a sol-gel method, and the light-scattering properties have been investigated by means of coherent backscattering. Three-dimensionally interconnected macroporous morphology is obtained by inducing the phase separation parallel to the sol-gel transition. The crystal structure of $\mathrm{TiO}_{2}$ gel is transformed from anatase to rutile through the heat treatment above $900{ }^{\circ} \mathrm{C}$, while the macroporous morphology remains unchanged. We show that the rutile-type $\mathrm{TiO}_{2}$-based macropurous monoliths are strongly scattering media for visible light. (C) 2004 American Institute of Physics. [DOI: 10.1063/1.1823596]
\end{abstract}

When the dielectric constant of materials varies on a length scale of the order of the wavelength of light, the materials couple strongly with light. Such materials have an index of refraction that can be either periodic or random in space, exhibiting interesting fundamental properties such as photon localization. Recently, considerable attention has been paid to the ordered and disordered dielectric systems because of their potential photonic applications. ${ }^{1}$

To achieve the photon localization in a disordered dielectric medium, light should be elastically scattered, meaning that light absorption is negligibly small. Also, the material must extremely strongly scatter light. The strength of scattering is largest for light having the wavelength $\lambda$ comparable to the size and spatial separation of the randomly distributed scatterers, and increases with an increase in refractive-index contrast $\Delta n$. So far, most studies on disordered dielectric media have been performed for fine particles, including powders and colloidal suspensions, and the scattering properties have been controlled by changing the density and size of particles. However, monolithic structures are favored rather than fine particles for practical use.

Pore formation is a very promising technique not only for obtaining monolithic scattering media but also for tailoring the scattering strength. Lagendijk and co-workers ${ }^{2}$ have induced macroporous morphology in high-refractive-index semiconductor gallium phosphide $(\mathrm{GaP})$ using an electrochemical etching, and demonstrated the strong scattering without optical absorption in the red part of the visible spectrum. They also showed that the scattering strength of the macroporous $\mathrm{GaP}$ can be tuned in a wide range, depending on the density and size of pores. ${ }^{3}$ On the other hand, we have fabricated macroporous monoliths in silica $\left(\mathrm{SiO}_{2}\right)$-based solgel systems, and investigated the light-scattering properties. ${ }^{4}$ The macroporous morphology is formed via the development of a transient structure of phase separation induced by the hydrolysis and polycondensation of alkoxysilane and the subsequent freezing of the structure by the sol-gel transition. ${ }^{5}$ Although in these systems the tunability of scattering strength was feasible with the control of the size and density

${ }^{a)}$ Electronic mail: koji@ collon1.kuic.kyoto-u.ac.jp of pores, the scattering strength was weak because of the low refractive index of $\mathrm{SiO}_{2}$ skeleton.

Since rutile-type titania $\left(\mathrm{TiO}_{2}\right)$ has a high refractive index of $n \sim 2.7$ and very low absorption in visible spectrum, if the pore formation is possible for $\mathrm{TiO}_{2}$, strongly scattering and weakly absorbing media that bring about photon localization would be realized in the whole visible regions. In spite of this advantage, few works have been performed on porous $\mathrm{TiO}_{2}$. One reason for this fact is the difficulty in preparing porous $\mathrm{TiO}_{2}$; in the conventional sol-gel system derived from titanium alkoxide, the structural development is hard to be controlled in the course of the hydrolysis and polycondensation because of the rapid polymerization reaction. More recently, we developed a route toward synthesizing macroporous $\mathrm{TiO}_{2}$ monoliths using the sol-gel method including phase separation. ${ }^{6}$ Fabrication is accomplished by the use of aqueous titania colloid instead of highly reactive titanium alkoxide. The aggregation and gelation of titania colloid are controlled by the $p \mathrm{H}$ increase due to the hydrolysis of formamide, while the phase separation is induced by an addition of poly(ethylene oxide) to the reaction mixture.

In this letter, it is shown that rutile-type $\mathrm{TiO}_{2}$-based macroporous monoliths that strongly scatter visible light can be obtained very simply by calcination of the porous gels derived from titania colloid. A characteristic feature of the present fabrication method is that the size and volume fraction of pores can be controlled by the composition of starting solutions and/or the reaction temperature. The tunability of the scattering strength through the change in the density and size of pores is also demonstrated.

The titania source employed in our experiments was aqueous dispersion of titania colloid having the anatase-type structure and an average size of about $7 \mathrm{~nm}$. Poly(ethylene oxide) (PEO) with an average molecular weight of 100000 was used as the polymer component to induce the phase separation. Formamide and $60 \mathrm{wt} \%$ aqueous solution of nitric acid were utilized as the solvents to control the aggregation and gelation of titania colloid. The calculated starting compositions are listed in Table I along with the reaction temperature for gelation. Gel samples were prepared by mixing titania colloid and formamide with the aqueous solution of nitric acid containing PEO. After stirring for $10 \mathrm{~min}$, the 
TABLE I. The calculated starting compositions and the gelation temperature $T_{g}$ of samples. Average pore diameter $d$, porosity $\phi$, and transport mean free path $l$ are also shown for the fully sintered samples heat-treated at $1200{ }^{\circ} \mathrm{C}$.

Content (unit:g)

\begin{tabular}{|c|c|c|c|c|c|c|c|c|c|}
\hline \multirow[b]{2}{*}{ Sample } & \multicolumn{5}{|c|}{ Content (unit:g) } & \multirow[b]{2}{*}{$\begin{array}{c}T_{g} \\
\left({ }^{\circ} \mathrm{C}\right)\end{array}$} & \multirow[b]{2}{*}{$\begin{array}{c}d \\
(\mu \mathrm{m})\end{array}$} & \multirow[b]{2}{*}{$\begin{array}{c}\phi \\
(\%)^{\mathrm{a}}\end{array}$} & \multirow[b]{2}{*}{$l(\mu \mathrm{m})$} \\
\hline & $\mathrm{TiO}_{2}$ & PEO & $\begin{array}{l}\text { Form- } \\
\text { amide }\end{array}$ & $\mathrm{HNO}_{3}$ & $\mathrm{H}_{2} \mathrm{O}$ & & & & \\
\hline (A) & 1.976 & 0.1 & 1.6 & 0.183 & 5.834 & 40 & 0.91 & 56.5 & 0.66 \\
\hline (B) & 1.976 & 0.1 & 1.6 & 0.290 & 9.277 & 40 & 1.46 & 64.5 & 0.66 \\
\hline (C) & 1.976 & 0.1 & 1.6 & 0.479 & 14.17 & 60 & 0.76 & 67.0 & 0.46 \\
\hline
\end{tabular}

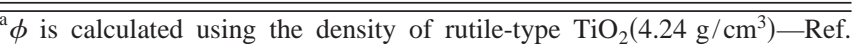
15-and the cumulative pore volume as shown in Fig. 2.

mixed solution was poured into a polystyrene test tube. Gelation was carried out at 40 or $60{ }^{\circ} \mathrm{C}$ in the sealed test tube. The wet gel thus obtained was aged for $24 \mathrm{~h}$ and subjected to the solvent exchange with 2-methyl-2-propanol. The solvent exchange was repeated five times. The resultant wet gel was freeze-dried to obtain the porous gel. In some cases, the dried gel was heat-treated at various temperatures of $500-1200{ }^{\circ} \mathrm{C}$ for $1 \mathrm{~h}$ in air.

Figure 1 depicts scanning electron microscope (SEM) images for sample (A). The morphology of interconnected structure is observed for the dried gel without heat treatment as shown in Fig. 1(a), indicating the formation of phaseseparated structures at the stage of wet gel. The phase separation probably proceeds via the spinodal decomposition. ${ }^{6}$ In the present system containing PEO, the phase separation is driven by a repulsive interaction between solvents and PEO adsorbed on colloidal $\mathrm{TiO}_{2} .{ }^{6}$ Hence, the reacting solution separates into two phases; one is rich in solvents and the other rich in $\mathrm{PEO}$ and $\mathrm{TiO}_{2}$. Bicontinuous structure, in which both separated phases are interconnected in threedimensional space, is obtained when the phase separation and gelation concur to fix the transient structure of phase separation. On drying, the phase with $\mathrm{PEO}$ and $\mathrm{TiO}_{2}$ becomes a gel skeleton, and the solvent phase turns into macropores that serve as throughpores.

The macroporous morphology is maintained even after the heat treatment at $1200{ }^{\circ} \mathrm{C}$ [see Fig. 1(b)]. We measured the pore size distribution using a mercury porosimetry for $\mathrm{TiO}_{2}$ gels heat-treated at $1200{ }^{\circ} \mathrm{C}$. The result is shown in Fig. 2, where the sharp distribution characteristic of the spinodal decomposition is obtained in all the samples. It is found that the pore size and pore volume vary with the starting composition and/or the reaction temperature. The average pore size and porosity deduced from these data are also summarized in Table I. A porosity as high as $67 \%$ is obtained in sample (C) prepared from the starting solution containing a
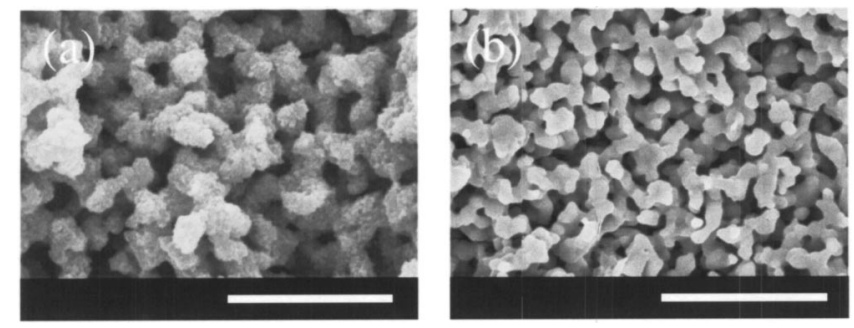

FIG. 1. SEM images of $\mathrm{TiO}_{2}$ gels prepared from the starting composition (A): (a) as-made dried gel (without heat treatment); (b) the gel heat-treated

the $\mathrm{TiO}_{2}$ skeleton and pore (air), which is caused both by the
at $120{ }^{\circ} \mathrm{C}$. Bars $=10 \mu \mathrm{m}$.
Downloaded 04 Mar 2008 to 130.54 .110 .22 . Redistribution subject to AlP license or copyright; see http://apl.aip.org/apl/copyright.jsp

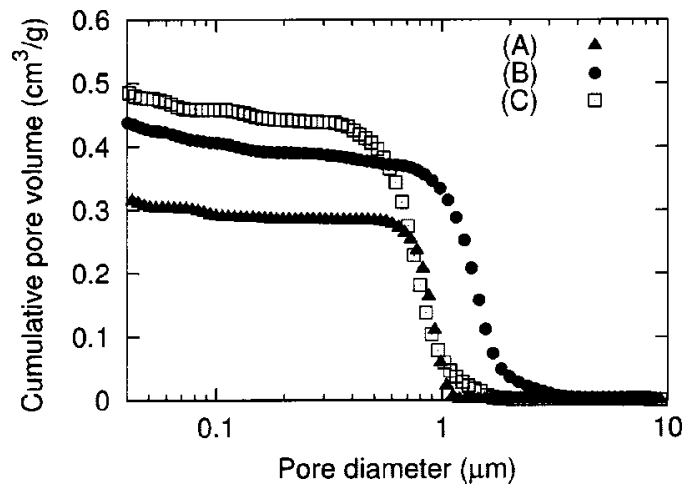

FIG. 2. Pore size distributions of $\mathrm{TiO}_{2}$ gels heat-treated at $1200{ }^{\circ} \mathrm{C}$.

large amount of solvents. As mentioned earlier, the solventrich phase induced by phase separation changes into macropores after drying, so that the volume fraction of pores depends on the solvent fraction in the starting solution. On the other hand, the pore size of sample $(\mathrm{C})$ is the smallest among the present samples. This is mainly because the gelation temperature $\left(\sim 60^{\circ} \mathrm{C}\right)$ is higher compared to that in samples (A) and (B). When the gelation temperature is higher, the $p \mathrm{H}$ in the reaction solution increases more rapidly to accelerate the aggregation and gelation of colloid. The transient structures of finer bicontinuous phases are frozen by the earlier gelation relative to the onset of phase separation, with smaller pores being left behind after drying. More systematic studies are now under investigation to clarify the relationship between the gel morphology and the starting composition or the reaction temperature.

Figure 3(a) shows the variation of $x$-ray diffraction (XRD) patterns with heat-treatment temperature for sample (B). All the diffraction lines for the dried gel without heat treatment (as-made gel) can be assigned to anatase-type $\mathrm{TiO}_{2}$. The broadening of diffraction peaks indicates the smallness of crystallite size. The crystallite size estimated by Scherrer's equation is about $10 \mathrm{~nm}$, which is consistent with the primary particle size of $\mathrm{TiO}_{2}$ colloid used as the starting materials. The skeleton of as-made gel thus consists of the aggregate of nanoparticles and is easily broken due to the low connectivity. As the heat-treatment temperature is raised, the gel skeleton is sintered into the fully dense body. In addition, the anatase phase is completely transformed into the rutile phase during the heat treatment above $900{ }^{\circ} \mathrm{C}$.

Light-scattering properties have been characterized using coherent backscattering (CBS) measurements. CBS is observed as an increase in the reflected intensity from a medium at the exact backscattering direction as a consequence of the interference of waves propagating along time-reversed optical paths. ${ }^{7-10}$ The CBS yields a cone in the plot of the backscattered intensity versus the scattering angle, and the full width of half maximum of the cone is inversely proportional to transport mean free path $l,{ }^{10}$ which is the average length required to randomize the direction of light propagation by scattering. Figure 3(b) shows the heat-treatment temperature dependence of CBS cone for sample (B). The measurements were performed using an $\operatorname{Ar}^{+}$laser $(\lambda=488 \mathrm{~nm})$. The CBS cone broadens as the heat-treatment temperature becomes higher, indicating stronger scattering or smaller $l$. The efficient scattering for the sample heat-treated at $900{ }^{\circ} \mathrm{C}$ is ascribable to the high refractive-index contrast $\Delta n$ between the $\mathrm{TiO}_{2}$ skeleton and pore (air), which is caused both by the 

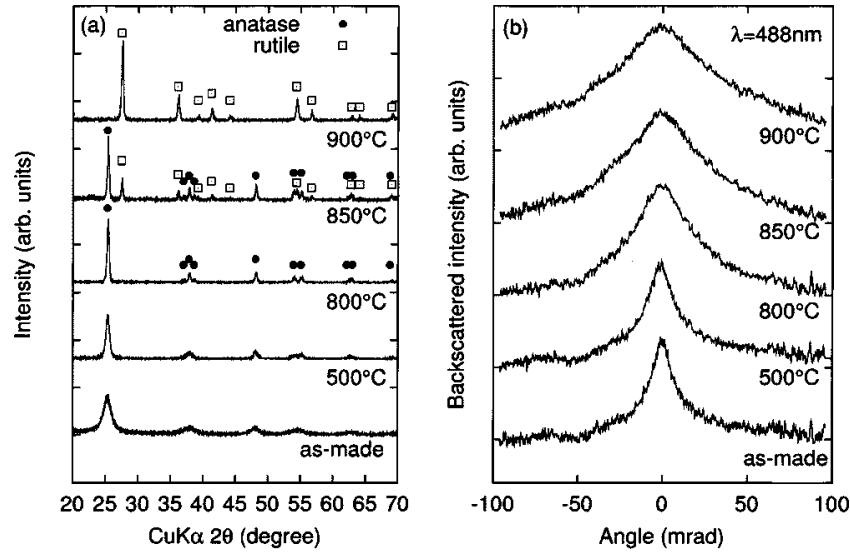

FIG. 3. (a) Variation of XRD patterns with heat-treatment temperature for sample (B). (b) Backscattered intensity normalized to the diffuse background as a function of scattering angle for sample (B) heat-treated at varied temperatures.

sintering into fully dense skeleton and by the transformation into the rutile phase whose refractive index is higher than that of the anatase phase.

The feasibility of controlling the pore size and the pore volume, as shown in Fig. 2, is useful for tuning the scattering strength. Figure 4 shows CBS results for $\mathrm{TiO}_{2}$ gels heattreated at $1200{ }^{\circ} \mathrm{C}$. For comparison, a typical CBS result is also shown for the macroporous $\mathrm{SiO}_{2}$ prepared by the alkoxy-derived sol-gel process including phase separation as we reported previously. ${ }^{4}$ We obtained $l$ from the fit of diffusion theory ${ }^{10}$ to the CBS data, as indicated by solid curves in Fig. 4. The value of $l$ for the $\mathrm{SiO}_{2}$ system is about $2.2 \mu \mathrm{m}$, whereas the broader CBS cones for $\mathrm{TiO}_{2}$ systems manifest smaller $l$ or stronger scattering. Especially, sample (C) is the strongest-scattering medium, and $l$ becomes smaller than $\lambda(=488 \mathrm{~nm})$ (see Table I). The transport mean free path is expressed as $l=1 / \rho \sigma$, where $\rho$ is the density of scatterers, and $\sigma$ is the transport cross section. ${ }^{11}$ According to the Miescattering theory, ${ }^{12} \sigma$ is largest when the size of scatterers is comparable to $\lambda$ and increases with an increase in $\Delta n$. This is true of spherical or cylindrical scatterers. Although the interconnected morphology obscures this description, the relatively short $l$ of sample (C) can be qualitatively interpreted

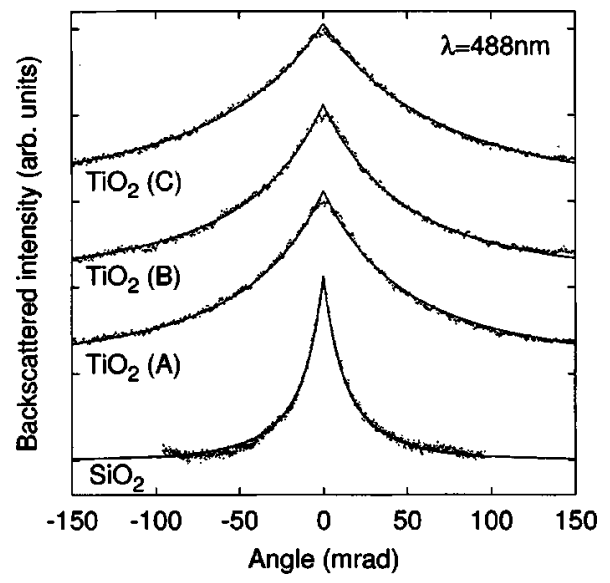

FIG. 4. Backscattered intensity normalized to the diffuse background as a function of scattering angle for $\mathrm{TiO}_{2}$ gels heat-treated at $1200{ }^{\circ} \mathrm{C}$. A typical CBS result for the macroporous $\mathrm{SiO}_{2}$ gel prepared by the sol-gel process including phase separation is also shown for comparison (see Ref. 4). The solid curves represent theoretical fits from diffusion approximation (see Ref. 10). by considering the pore size and pore density in addition to the high $\Delta n$, since the pores can be regarded as scatterers in the present systems. As seen from Table I, the pore size is smallest for sample (C), while the porosity is the largest. The small pore size and large porosity give rise to the short distance between pores, leading to the high pore density $\rho$. Also, the pore size of sample (C) is close to the wavelength of $488 \mathrm{~nm}$, so that the transport cross section $\sigma$ becomes large. Consequently, the large $\rho$ and $\sigma$ are responsible for the short $l$ or strong scattering.

The approach to synthesize strongly scattering $\mathrm{TiO}_{2}$ monoliths as presented here is quite simple and easy, so that it is expected that they find some application fields in photonics. When emission sources, such as organic dye and semiconductor nanoparticles, are combined with the strong scattering, stimulated emission may occur by the spontaneous feedback of emitted photons through the interference of multiply scattered light, resulting in mirrorless laser action, so-called a random laser. ${ }^{13}$ Also, Wiersma and Cavalieri ${ }^{14}$ prepared glass powders infiltrated with liquid crystal and laser dye, and demonstrated the random laser that can be controlled externally via temperature. In the present case, this will be readily achieved by introducing dye-dissolved liquid crystal into throughpores. Besides the photonic applications, macroporous $\mathrm{TiO}_{2}$ monoliths are widely applicable to electrode materials, gas sensors, and chromatographies, because the increased surface area and chemical accessibility associated with throughpores offer distinct advantage.

In conclusion, we have shown the strong scattering of visible light in rutile-type $\mathrm{TiO}_{2}$-based macroporous monoliths. The macroporous morphology can be successfully formed by inducing the phase separation in the sol-gel process started from aqueous dispersion of $\mathrm{TiO}_{2}$ colloid in the presence of PEO. For fully sintered samples, the transport mean free path for visible light decreases down to the order of the wavelength. The scattering strength is tuned by the composition of starting solutions and/or the reaction temperature. We expect that this type of porous materials holds extended applicability in various technological fields.

${ }^{1}$ Photonic Crystals and Light Localization in the 21st Century, edited by C. M. Soukoulis (Kluwer, Dordrecht, 2001).

${ }^{2}$ F. J. P. Schuurmans, D. Vanmaekelbergh, J. van de Lagemaat, and A. Lagendijk, Science 284, 141 (1999); F. J. P. Schuurmans, M. Megens, D. Vanmaekelbergh, and A. Lagendijk, Phys. Rev. Lett. 83, 2183 (1999).

${ }^{3}$ J. G. Rivas, A. Lagendijk, R. W. Tjerkstra, D. Vanmaekelbergh, and J. J. Kelly, Appl. Phys. Lett. 80, 4498 (2002).

${ }^{4}$ S. Murai, K. Fujita, K. Nakanishi, and K. Hirao, Jpn. J. Appl. Phys., Part 143,5359 (2004)

${ }^{5}$ K. Nakanishi, J. Porous Mater. 4, 67 (1997).

${ }^{6}$ J. Konishi, K. Fujita, K. Nakanishi, and K. Hirao, Mater. Res. Soc. Symp. Proc. 788, 391 (2004).

${ }^{7}$ Y. Kuga and A. Ishimaru, J. Opt. Soc. Am. A 1, 831 (1984).

${ }^{8}$ M. P. van Albada and A. Lagendijk, Phys. Rev. Lett. 55, 2692 (1985).

${ }^{9}$ P. Wolf and G. Maret, Phys. Rev. Lett. 55, 2696 (1985).

${ }^{10}$ E. Akkermans, P. Wolf, and R. Maynard, Phys. Rev. Lett. 56, 1471 (1986).

${ }^{11}$ A. Ishimaru, Wave Propagation and Scattering in Random Media (Academic, New York, 1978), Vol. 1, p. 178.

${ }^{12}$ H. C. van de Hulst, Light Scattering by Small Particles (Dover, New York, 1957).

${ }^{13}$ N. M. Lawandy, R. M. Balachandran, A. S. L. Gomes, and E. Sauvain, Nature (London) 368, 436 (1994).

${ }^{14}$ D. S. Wiersma and S. Cavalieri, Nature (London) 414, 708 (2001).

${ }^{15}$ The Oxide Handbook, edited by G. V. Samsonov (Plenum, New York, 1973), p. 34. 